Commun. Fac. Sci. Univ. Ank. Ser. A1 Math. Stat.

Volume 68, Number 2, Pages 22982312(2019)

DOI: $10.31801 /$ cfsuasmas.000000

ISSN 1303-5991 E-ISSN 2618-6470

http://communications.science.ankara.edu.tr/index.php?series=A1

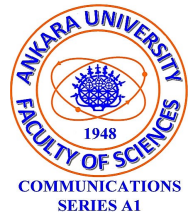

\title{
[1,2]-COMPLEMENTARY CONNECTED DOMINATION NUMBER OF GRAPHS-III
}

\author{
G.MAHADEVAN AND K.RENUKA
}

\begin{abstract}
A set $S \subseteq V(G)$ in a graph $\mathrm{G}$ is said to be [1,2]-complementary connected dominating set if for every vertex $v \in V-S, 1 \leq|N(v) \cap S| \leq 2$ and $\langle V-S\rangle$ is connected. The minimum cardinality of $[1,2]$-complementary connected dominating set is called $[1,2]$-complementary connected domination number and is denoted by $\gamma_{[1,2] c c}(G)$. In this paper, we investigate 3 -regular graphs on twelve vertices for which $\gamma_{[1,2] c c}(G)=\chi(G)=3$.
\end{abstract}

\section{INTRODUCTION}

Let $G(V, E)$ be simple and connected graph. For graph theoretic terminology we refer to Chartrand and Lesniak [1] and Haynes et.al [2]. In [6], V.R.Kulli and B.Janakiraman introduced the concept of nonsplit domination number of graph and characterized its bounds. In [3], Mustapha Chellali et.al, first studied the concept of $[1,2]-$ sets. In [7, Xiaojing Yang and Baoyindureng $\mathrm{Wu}$, extended to the study of this parameter. In [4, 5], G.Mahadevan et.al, introduced the concept of $[1,2]$-complementary connected domination and investigate 3 -regular graphs of order $n \leq 10$, whose [1,2]-complementary connected domination number equals chromatic number equals three. In this paper, we investigate 3 -regular graphs on twelve vertices for which $\gamma_{[1,2] c c}(G)=\chi(G)=3$.

\section{3-REgUlAR GRAPHS ON TWELVE VERTICES}

Let $\mathrm{G}$ be a connected cubic graph on twelve vertices for which $\chi(G)=\gamma_{[1,2] c c}(G)=$ 3. Let $S=\{x, y, z\}$ be a $[1,2] c c$-set. Since $G$ is cubic, clearly $\langle S\rangle \neq K_{3}, K_{2} \cup$

Received by the editors: February 05, 2018; Accepted: June 25, 2018.

2010 Mathematics Subject Classification. 05C69.

Key words and phrases. Complementary connected domination, [1,2]-sets, [1,2]-domination, $[1,2]$-complementary connected domination.

Submitted via International Conference on Current Scenario in Pure and Applied Mathematics [ICCSPAM 2018].

This research work was supported by Departmental Special Assistance, University Grants Commission, New Delhi and UGC-BSR Research fellowship in Mathematical Sciences- 2014-2015. 
$K_{1}, P_{3}$. Hence $\left\langle S>=\bar{K}_{3}\right.$. Let $S_{1}=\left\{v_{1}, v_{2}, v_{3}\right\}, S_{2}=\left\{v_{4}, v_{5}, v_{6}\right\}$ and $S_{3}=$ $\left\{v_{7}, v_{8}, v_{9}\right\}$. The following are only possible cases $\left\langle S_{i}\right\rangle$, where $1 \leq i \leq 3$. Let $<S_{1}>=<S_{2}>=<S_{3}>=P_{3} ;<S_{1}>=<S_{2}>=P_{3},<S_{3}>=K_{2} \cup K_{1}$; $<S_{1}>=<S_{2}>=P_{3},<S_{3}>=\bar{K}_{3} ;<S_{1}>=P_{3},<S_{2}>=<S_{3}>=K_{2} \cup K_{1}$; $<S_{1}>=P_{3},<S_{2}>=K_{2} \cup K_{1},<S_{3}>=\bar{K}_{3} ;<S_{1}>=P_{3},<S_{2}>=<S_{3}>=\bar{K}_{3}$; $<S_{1}>=<S_{2}>=<S_{3}>=K_{2} \cup K_{1} ;<S_{1}>=<S_{2}>=K_{2} \cup K_{1},<S_{3}>=K_{3}$; $<S_{1}>=K_{2} \cup K_{1},<S_{2}>=<S_{3}>=\bar{K}_{3} ;<S_{1}>=<S_{2}>=<S_{3}>=\bar{K}_{3}$.

Graphs $G_{i}$, where $1 \leq i \leq 32$

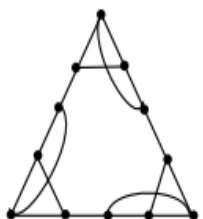

$G_{1}$

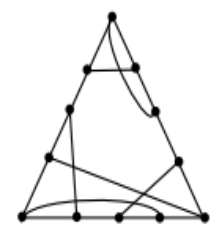

$G_{5}$

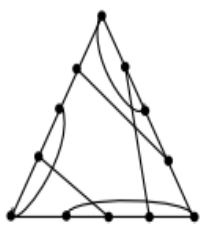

$G_{9}$

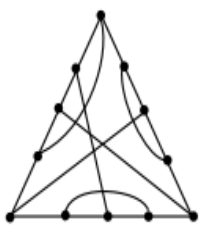

$G_{13}$

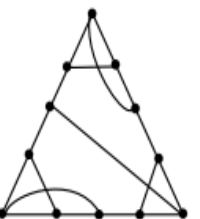

$G_{2}$

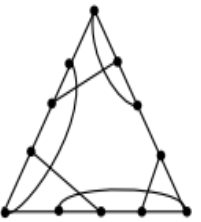

$G_{6}$

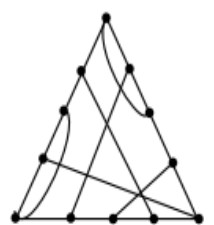

$G_{10}$

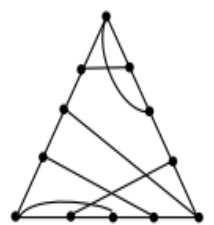

$G_{14}$

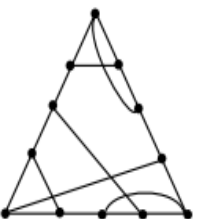

$G_{3}$

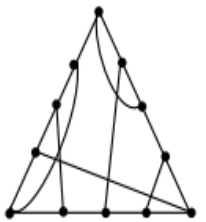

$G_{7}$

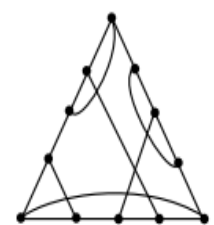

$G_{11}$

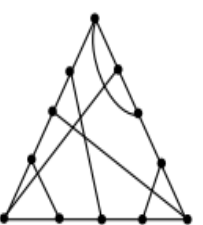

$G_{15}$

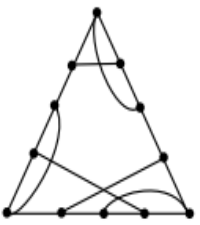

$G_{4}$

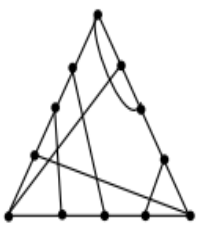

Gs

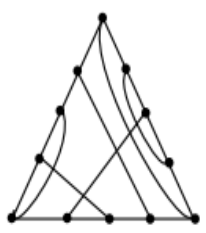

$G_{12}$

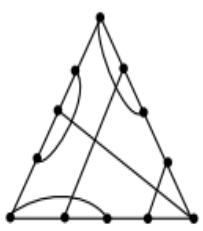

$G_{16}$ 


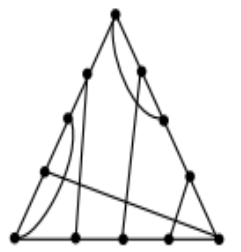

$G_{17}$

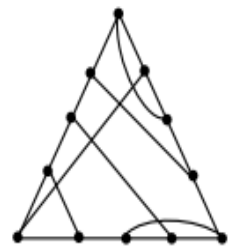

$G_{21}$

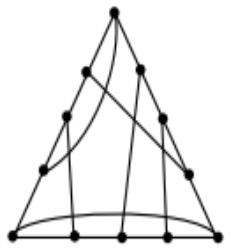

$G_{25}$

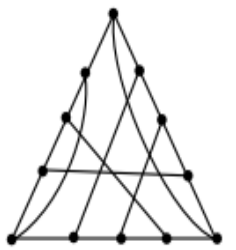

$G_{2}$

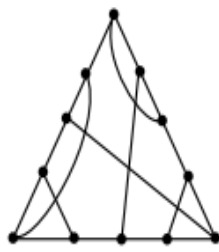

$G_{18}$

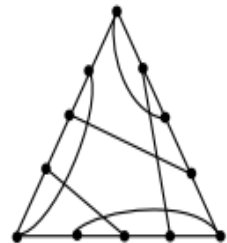

$G_{22}$

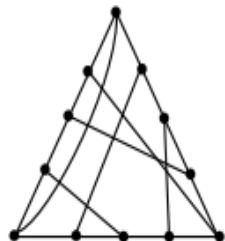

$G_{26}$

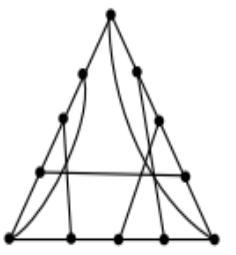

$G_{30}$

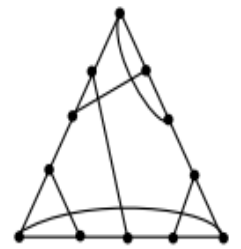

$G_{19}$

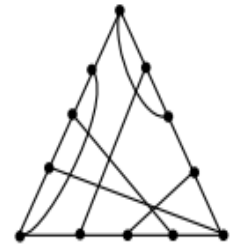

$G_{23}$

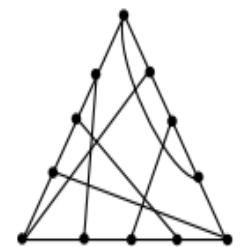

$G_{27}$

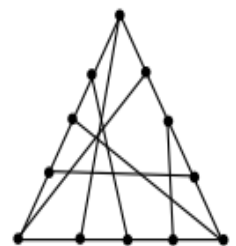

$G_{31}$

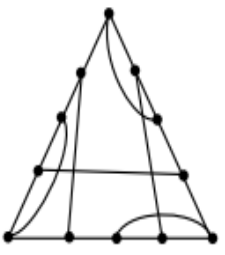

$G_{20}$

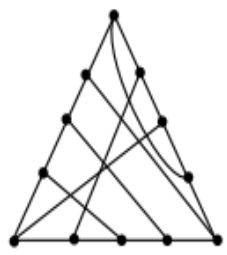

$G_{24}$

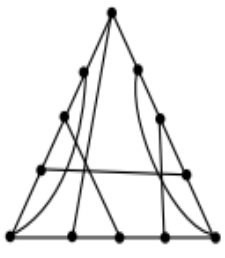

$G_{28}$

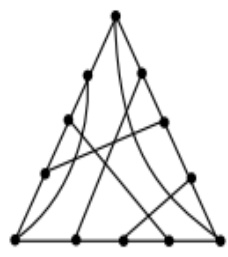

$G_{32}$

Prepositon 2.1. If $<S>=\bar{K}_{3}$ and $<S_{1}>=<S_{2}>=<S_{3}>=P_{3}$, then $G \cong G_{1}$

Proof. Let $<S_{1}>=P_{3}=\left(v_{1}, v_{2}, v_{3}\right),<S_{2}>=P_{3}=\left(v_{4}, v_{5}, v_{6}\right)$ and $<S_{3}>=$ $P_{3}=\left(v_{7}, v_{8}, v_{9}\right)$. Let $v_{1}$ be adjacent to any one of $\left\{v_{4}, v_{6}, v_{7}, v_{9}\right\}$. Without loss of generality, let $v_{1}$ be adjacent to $v_{4}$. Now $v_{3}$ is adjacent to $v_{6}$ or $v_{7}$ (or equivalently to $v_{9}$ ). If $v_{3}$ is adjacent to $v_{6}$, then no new graph exists. If $v_{3}$ is adjacent to $v_{7}$, then $G \cong G_{1}$. 
Prepositon 2.2. If $<S>=\bar{K}_{3}$ and $<S_{1}>=<S_{2}>=P_{3}$ and $<S_{3}>=K_{2} \cup K_{1}$, then $G \cong G_{2}$

Proof. Let $<S_{1}>=P_{3}=\left(v_{1}, v_{2}, v_{3}\right),<S_{2}>=P_{3}=\left(v_{4}, v_{5}, v_{6}\right)$ and $<S_{3}>=$ $P_{3}=\left(v_{7}, v_{8}, v_{9}\right)$, where $v_{7} v_{8} \in E\left(S_{3}\right)$. Let $v_{1}$ be adjacent to any one of $\left\{v_{4}, v_{6}\right\}$ or any one of $\left\{v_{7}, v_{8}\right\}$ or $v_{9}$.

Case $1 v_{1} v_{4} \in E(G)$

Let $v_{3}$ be adjacent to $v_{6}$ or $v_{7}$ (or equivalently to $v_{8}$ ) or $v_{9}$. If $v_{3}$ is adjacent to $v_{6}$, then no graph exists.

If $v_{3}$ is adjacent to $v_{7}$, then either $v_{6}$ is adjacent to $v_{8}$ or $v_{9}$. If $v_{6}$ is adjacent to $v_{8}$, then no new graph exists. If $v_{6}$ is adjacent to $v_{9}$, then no graph exists.

If $v_{3}$ is adjacent to $v_{9}$, then either $v_{6}$ is adjacent to any one of $\left\{v_{7}, v_{8}, v_{9}\right\}$. If $v_{6}$ is adjacent to $v_{7}$ or $v_{8}$ or $v_{9}$, then no graph exists.

Case $2 v_{1} v_{7} \in E(G)$

Let $v_{3}$ be adjacent to any one of $\left\{v_{4}, v_{6}\right\}$ or $v_{8}$ or $v_{9}$. Let $v_{3}$ be adjacent to $v_{4}$. Then $v_{6}$ is adjacent to any one of $v_{8}$ or $v_{9}$ and hence no graph exists.

Let $v_{3}$ be adjacent to $v_{8}$. Then $v_{9}$ is adjacent to $v_{4}$ and $v_{6}$. In this case, $\langle V-S\rangle$ is disconnected and hence no graph exists.

Let $v_{3}$ be adjacent to $v_{9}$. Then $v_{4}$ is adjacent to $v_{8}$ or $v_{9}$. If $v_{4}$ is adjacent to $v_{8}$, then $v_{9}$ is adjacent to $v_{6}$ and hence $G \cong G_{2}$. If $v_{4}$ is adjacent to $v_{9}$, then $v_{6}$ is adjacent to $v_{8}$ and hence $G \cong G_{2}$.

Case $3 v_{1} v_{9} \in E(G)$

Let $v_{3}$ be adjacent to any one of $\left\{v_{4}, v_{6}\right\}$ or any one of $\left\{v_{7}, v_{8}\right\}$ or $v_{9}$. If $v_{3}$ is adjacent to $v_{4}$, then no new graph exists.

If $v_{3}$ is adjacent to $v_{7}$, then $v_{8}$ is adjacent to $v_{4}$ (or eqivalently to $v_{6}$ ). If $v_{8}$ is adjacent to $v_{6}$, then $v_{9}$ is adjacent to $v_{4}$, so that $G \cong G_{2}$.

If $v_{3}$ is adjacent to $v_{9}$, then $v_{7}$ is adjacent to $v_{4}$ and $v_{8}$ is adjacent to $v_{6}$. In this case $\langle V-S\rangle$ is disconnected and hence no graph exists.

Prepositon 2.3. If $<S>=\bar{K}_{3}$ and $<S_{1}>=<S_{2}>=P_{3}$ and $<S_{3}>=\bar{K}_{3}$, then no graph exists.

Proof. Let $<S_{1}>=P_{3}=\left(v_{1}, v_{2}, v_{3}\right),<S_{2}>=P_{3}=\left(v_{4}, v_{5}, v_{6}\right)$ and $<S_{3}>=$ $\bar{K}_{3}=\left(v_{7}, v_{8}, v_{9}\right)$. Let $v_{1}$ be adjacent to any one of $\left\{v_{4}, v_{6}\right\}$ or $\left\{v_{7}, v_{8}, v_{9}\right\}$

Case 1 Let $v_{1}$ be adjacent to $v_{4}$. Since G is cubic, $v_{3}$ cannot be adjacent to $v_{6}$ and hence $v_{3}$ is adjacent to any one of $\left\{v_{7}, v_{8}, v_{9}\right\}$. If $v_{3}$ is adjacent to $v_{7}$, then no new graph exists.

Case 2 Let $v_{1}$ be adjacent to $v_{7}$ and $v_{3}$ is adjacent to any one of $\left\{v_{4}, v_{6}\right\}$ or any one of $\left\{v_{8}, v_{9}\right\}$ or $v_{7}$. In all the above cases, no graph exists.

Prepositon 2.4. If $\left\langle S>=\bar{K}_{3}\right.$ and $<S_{1}>=P_{3}$ and $<S_{2}>=<S_{3}>=K_{2} \cup K_{1}$, then $G \cong G_{i}$, where $i=3$, 4 . 
Proof. Let $<S_{1}>=P_{3}=\left(v_{1}, v_{2}, v_{3}\right),<S_{2}>=K_{2} \cup K_{1}=\left(v_{4}, v_{5}, v_{6}\right)$ and $<$ $S_{3}>=K_{2} \cup K_{1}=\left(v_{7}, v_{8}, v_{9}\right)$, where $v_{4} v_{5}, v_{7} v_{8} \in E(G)$. Let $v_{1}$ be adjacent to $\left\{v_{4}, v_{5}, v_{7}, v_{8}\right\}$ or $\left\{v_{6}, v_{9}\right\}$.

Case $1 v_{1} v_{4} \in E(G)$.

In this case, $v_{3}$ must be adjacent to $v_{5}$ or $v_{6},\left\{v_{7}, v_{8}\right\}$ or $v_{9}$.

Let $v_{3}$ be adjacent to $v_{5}$. Then $v_{6}$ is adjacent to either $\left\{v_{7}\right.$ and $\left.v_{8}\right\}$ or $\left\{v_{7}\right.$ and $\left.v_{9}\right\}$. If $v_{6}$ is adjacent to $v_{7}$ and $v_{8}$, then no graph exists. If $v_{6}$ is adjacent to $v_{7}$ and $v_{9}$, then no graph exists.

If $v_{3}$ is adjacent to $v_{6}$, then $v_{5}$ is adjacent to either $v_{9}$ or any one of $\left\{v_{7}, v_{8}\right\}$. If $v_{5}$ is adjacent to $v_{9}$, then no graph exists. If $v_{5}$ is adjacent to $v_{7}$, then no graph exists.

Case $2 v_{1} v_{6} \in E(G)$.

Let $v_{3}$ be adjacent to either $v_{9}$ or any one of $\left\{v_{4}, v_{5}\right\}$ or any one of $\left\{v_{7}, v_{8}\right\}$.

If $v_{3}$ is adjacent to $v_{9}$, then $v_{4}$ is adjacent to any one of $\left\{v_{7}, v_{8}\right\}$ or $v_{9}$. If $v_{4}$ is adjacent to $v_{9}$ and $v_{5}$ is adjacent to $v_{7}$, then $v_{6}$ is adjacent to $v_{8}$ and hence $G \cong G_{3}$. If $v_{4}$ is adjacent to $v_{7}$, then $v_{5}$ is adjacent to $v_{8}$ or $v_{9}$. If $v_{5}$ is adjacent to $v_{8}$, and $v_{6}$ is adjacent to $v_{9}$, then $\langle V-S\rangle$ is disconnected. If $v_{5}$ is adjacent to $v_{9}$, and $v_{6}$ is adjacent to $v_{8}$, then $G \cong G_{3}$. Let $v_{4}$ be adjacent to $v_{7}$. Then $v_{5}$ is adjacent to $v_{8}$ or $v_{9}$. If $v_{5}$ is adjacent to $v_{8}$, then $v_{6}$ is adjacent to $v_{9}$ and $\langle V-S\rangle$ is disconnected. If $v_{5}$ is adjacent to $v_{9}$ and $v_{6}$ is adjacent to $v_{8}$, then $G \cong G_{3}$.

Let $v_{3}$ be adjacent to $v_{4}$. Then $v_{5}$ is adjacent to any one of $\left\{v_{7}, v_{8}\right\}$ or $v_{9}$. If $v_{5}$ is adjacent ot $v_{7}$, then $v_{6}$ is adjacent to $v_{8}$ or $v_{7}$. If $v_{6}$ is adjacent to $v_{8}$, then no graph exists. If $v_{5}$ is adjacent to $v_{9}$, then $v_{6}$ is adjacent to any one of $\left\{v_{7}, v_{8}\right\}$ or $v_{9}$. If $v_{6}$ is adjacent to $v_{7}$, then no graph exists. If $v_{6}$ is adjacent to $v_{9}$, then no graph exists.

Let $v_{3}$ be adjacent to $v_{7}$. Then $v_{4}$ is adjacent to $v_{8}$ or $v_{9}$. If $v_{4}$ is adjacent to $v_{8}$, then $v_{9}$ is adjacent to $v_{5}$ and $v_{6}$. Hence $G \cong G_{4}$. If $v_{4}$ is adjacent to $v_{9}$, then $v_{5}$ is adjacent to $v_{8}$ or $v_{9}$. Without loss of generality, let $v_{5}$ is adjacent to $v_{8}$, then $v_{6}$ is adjacent to $v_{9}$. Hence $G \cong G_{4}$. If $v_{5}$ is adjacent to $v_{9}$, then $v_{6}$ is adjacent to $v_{8}$. In this case $\langle V-S\rangle$ is disconnected and hence no graph exists.

Prepositon 2.5. If $\langle S\rangle=<S_{2}>=\bar{K}_{3}$ and $<S_{1}>=P_{3}$ and $<S_{3}>=K_{2} \cup K_{1}$, then $G \cong G_{14}$.

Proof. Let $\left\langle S_{1}\right\rangle=P_{3}=\left(v_{1}, v_{2}, v_{3}\right),\left\langle S_{2}\right\rangle=\bar{K}_{3}=\left(v_{4}, v_{5}, v_{6}\right)$ and $\left\langle S_{3}\right\rangle=$ $K_{2} \cup K_{1}=\left(v_{7}, v_{8}, v_{9}\right)$, where $v_{7} v_{8} \in E(G)$. Let $v_{1}$ be adjacent to any one of $\left\{v_{4}, v_{5}, v_{6}\right\}$ or $\left\{v_{7}, v_{8}\right\}$ or $v_{9}$.

Case 1 Let $v_{1}$ be adjacent to $v_{4}$. Then $v_{3}$ is adjacent to any one of $\left\{v_{5}, v_{6}\right\}$ or any one of $\left\{v_{7}, v_{8}\right\}$ or $v_{9}$ or $v_{4}$.

Let $v_{3}$ be adjacent to $v_{5}$. Then $v_{6}$ is adjacent to any one of $\left\{v_{7}, v_{9}\right\}$ or any one of $\left\{v_{9}, v_{7}\right\}$. If $v_{6}$ is adjacent to $v_{7}$ and $v_{8}$. Then $v_{9}$ is adjacent to $v_{4}$ and $v_{5}$. Hence $\langle V-S\rangle$ is disconnected and hence no graph exists. If $v_{6}$ is adjacent to $v_{7}$ and $v_{9}$, then $v_{8}$ is adjacent to $v_{4}$ and $v_{9}$ is adjacent to $v_{5}$. Hence $G \cong G_{14}$. 
Let $v_{3}$ be adjacent to $v_{7}$. Then $v_{8}$ is adjacent to any one of $v_{7}$ or $\left\{v_{5}, v_{6}\right\}$. If $v_{8}$ is adjacent to $v_{4}$, then $v_{9}$ must be adjacent to $v_{5}$ and $v_{6}$. Hence no graph exists. If $v_{8}$ is adjacent to $v_{5}$, then $v_{9}$ must be adjacent to $\left\{v_{4}, v_{5}\right\}$ or $\left\{v_{4}, v_{6}\right\}$. In both cases, no graph exists.

Let $v_{3}$ be adjacent to $v_{9}$. Then $v_{9}$ is adjacent to $v_{4}$ or any one of $\left\{v_{5}, v_{6}\right\}$. If $v_{9}$ is adjacent to $v_{4}$, then either $v_{5}$ or $v_{6}$ is adjacent to $v_{7}$ and $v_{8}$ or $v_{5}$ is adjacent to $v_{7}$ and $v_{6}$ is adjacent to $v_{8}$. In both cases no graph exists. If $v_{7}$ is adjacent to $v_{5}$ and $v_{8}$ is adjacent to $\left\{v_{4}\right.$ and $\left.v_{5}\right\}$ or $\left\{v_{5}\right.$ and $\left.v_{6}\right\}$. In both cases no graph exists.

Let $v_{3}$ be adjacent to $v_{4}$. Then $v_{9}$ is adjacent to $v_{5}$ and $v_{6}, v_{7}$ is adjacent to any one of $\left\{v_{5}, v_{6}\right\}$. In this case $\langle V-S\rangle$ is disconnected and hence no graph exists. Case 2 Let $v_{1}$ be adjacent to $v_{7}$. Then $v_{3}$ is adjacent to any one of $\left\{v_{4}, v_{5}, v_{6}\right\}$ or $v_{8}$ or $v_{9}$.

Let $v_{3}$ be adjacent to $v_{4}$. Then $v_{4}$ is adjacent to any one of $v_{8}$ or $v_{9}$. If $v_{4}$ is adjacent to $v_{8}$, then $v_{9}$ is adjacent to $v_{5}$ and $v_{6}$ and no graph exists. If $v_{4}$ is adjacent to $v_{9}$, then $v_{9}$ is adjacent to any one of $\left\{v_{5}, v_{6}\right\}$. If $v_{9}$ is adjacent to $v_{5}$ or $v_{6}$, then no new graph exists.

Let $v_{3}$ be adjacent to $v_{8}$. Then $v_{9}$ is adjacent to any two of $\left\{v_{4}, v_{5}, v_{6}\right\}$ and hence no new graph exists. Let $v_{3}$ be adjacent to $v_{9}$. Then no graph exists.

Case 3 Let $v_{1}$ be adjacent to $v_{9}$. Then $v_{3}$ is adjacent to any one of $\left\{v_{4}, v_{5}, v_{6}\right\}$ or any one of $\left\{v_{7}, v_{8}\right\}$ or $v_{9}$.

Let $v_{3}$ be adjacent to $v_{4}$. Then $v_{4}$ must be adjacent to any one of $\left\{v_{7}, v_{8}\right\}$ or $v_{9}$. In both cases, no graph exists.

Let $v_{3}$ be adjacent to $v_{7}$. Then $v_{4}$ is adjacent to $v_{8}$ and $v_{9}$. Hence no graph exists. Let $v_{3}$ be adjacent to $v_{9}$. In this case, $\langle V-S\rangle$ is disconnected and hence no graph exists.

Prepositon 2.6. If $<S>=\bar{K}_{3}$ and $<S_{1}>=P_{3}$ and $<S_{2}>=<S_{3}>=\bar{K}_{3}$, then $G \cong G_{5}$.

Proof. Let $<S_{1}>=P_{3}=\left(v_{1}, v_{2}, v_{3}\right),<S_{2}>=\bar{K}_{3}=\left(v_{4}, v_{5}, v_{6}\right)$ and $<S_{3}>=$ $\bar{K}_{3}=\left(v_{7}, v_{8}, v_{9}\right)$. Let $v_{1}$ be adjacent to any one of $\left\{v_{4}, v_{5}, v_{6}, v_{7}, v_{8}, v_{9}\right\}$. Without loss of generality, let $v_{1}$ be adjacent to $v_{4}$. Then $v_{3}$ is adjacent to any one of $\left\{v_{5}, v_{6}\right\}$ or any one of $\left\{v_{7}, v_{8}, v_{9}\right\}$ or $v_{4}$.

Case 1 Let $v_{3}$ be adjacent to $v_{4}$. Then $v_{5}$ is adjacent to any two of $\left\{v_{7}, v_{8}, v_{9}\right\}$. In this case, $\langle V-S\rangle$ is disconnected and hence no graph exists.

Case 2 Let $v_{3}$ be adjacent to $v_{5}$. Then $v_{4}$ is adjacent to any one of $\left\{v_{7}, v_{8}, v_{9}\right\}$. If $v_{4}$ is adjcent to $v_{7}$, then $v_{5}$ is adjacent to $v_{7}$ or $\left\{v_{8}, v_{9}\right\}$.

If $v_{5}$ is adjacent to $v_{7}$, then $v_{6}$ is adjacent to $v_{8}$ and $v_{9}$. Hence no graph exists. If $v_{5}$ is adjacent to $v_{8}$, then $v_{6}$ is adjacent to any one of $\left\{v_{7}, v_{8}\right\}$ or any one of $\left\{v_{7}, v_{9}\right\}$. In both cases, no graph exists.

Case 3 Let $v_{3}$ be adjacent to $v_{7}$. Then $v_{4}$ is adjacent to any one of $\left\{v_{8}, v_{9}\right\}$ or $v_{7}$.

Let $v_{4}$ be adjacent to $v_{8}$. Then $v_{5}$ is adjacent to any one of $\left\{v_{7}, v_{8}\right\}$ or any one of $\left\{v_{8}, v_{9}\right\}$. Let $v_{5}$ be adjacent to $v_{7}$ and $v_{8}$. Then $v_{6}$ is adjacent to $v_{9}$ and hence 
no graph exists. If $v_{5}$ is adjacent to $v_{7}$ and $v_{9}$, then $v_{6}$ is adjacent to $v_{8}$ and $v_{9}$. Hence $G \cong G_{5}$.

Prepositon 2.7. If $\left\langle S>=\bar{K}_{3}\right.$ and $<S_{1}>=<S_{2}>=K_{2} \cup K_{1}$ and $<S_{3}>=\bar{K}_{3}$, then $G \cong G_{i}$, where $6 \leq i \leq 13$.

Proof. Let $\left\langle S_{1}>=K_{2} \cup K_{1}=\left(v_{1}, v_{2}, v_{3}\right),\left\langle S_{2}>=K_{2} \cup K_{1}=\left(v_{4}, v_{5}, v_{6}\right)\right.\right.$ and $<S_{3}>=\bar{K}_{3}=\left(v_{7}, v_{8}, v_{9}\right)$. Let $v_{1}$ be adjacent to any one of $\left\{v_{4}, v_{5}\right\}$ or $v_{6}$ or any one of $\left\{v_{7}, v_{8}, v_{9}\right\}$.

Case 1 Let $v_{1}$ be adjacent to $v_{4}$. Then $v_{2}$ is adjacent to $v_{5}$ or $v_{6}$ or any one of $\left\{v_{7}, v_{8}, v_{9}\right\}$. If $v_{2}$ is adjacent to $v_{5}$, then $\langle V-S\rangle$ is disconnected and hence no graph exists in this case.

Subcase 1 Let $v_{2} v_{6} \in E(G)$

Let $v_{3}$ be adjacent to $\left\{v_{5}, v_{7}\right\}$ or $\left\{v_{6}, v_{7}\right\}$ or $\left\{v_{7}, v_{8}, v_{9}\right\}$. If $v_{3}$ is adjacent to $v_{5}$ and $v_{6}$. Then $\langle V-S\rangle$ is disconnected and hence no graph exists.

Let $v_{3}$ be adjacent to $v_{5}$ and $v_{7}$. Then $v_{6}$ is adjacent to any one of $\left\{v_{7}, v_{8}, v_{9}\right\}$ and hence no graph exists. If $v_{3}$ is adjacent to $v_{6}$ and $v_{4}, v_{5}$ is adjacent to any one of $\left\{v_{7}, v_{8}, v_{9}\right\}$, then no graph exists in this case.

Let $v_{3}$ be adjacent to $v_{7}$ and $v_{8}$. Then $v_{5}$ is adjacent to any one of $\left\{v_{7}, v_{8}\right\}$ or $v_{9}$. If $v_{5}$ is adjacent to $v_{7}$, then $v_{6}$ is adjacent to $v_{8}$. In both cases no graph exists.

If $v_{5}$ is adjacent to $v_{9}$, then $v_{6}$ is adjacent to any one of $\left\{v_{7}, v_{8}, v_{9}\right\}$. Hence no graph exists for this case.

Subcase 2 Let $v_{2} v_{7} \in E(G)$

Let $v_{3}$ be adjacent to both of $\left\{v_{5}, v_{6}\right\}$ or $\left\{v_{5}, v_{7}\right\}$ or $\left\{v_{5}, v_{8}\right\}$ or $\left\{v_{6}, v_{7}\right\}$ or $\left\{v_{6}, v_{8}\right\}$ or $\left\{v_{7}, v_{8}\right\}$ or $\left\{v_{8}, v_{9}\right\}$.

If $v_{3}$ is adjacent to $v_{5}$ and $v_{6}$, then $v_{6}$ is adjacent to any one of $\left\{v_{7}, v_{8}, v_{9}\right\}$. In this case, no graph exists. If $v_{3}$ is adjacent to $v_{5}$ and $v_{7}$, then $v_{6}$ is adjacent to any one of $v_{8}$ and $v_{9}$. Hence no graph exists.

If $v_{3}$ is adjacent to $v_{5}$ and $v_{8}$, then $v_{6}$ is adjacent to any two of $\left\{v_{7}, v_{8}, v_{9}\right\}$. Hence no graph exists. If $v_{3}$ is adjacent to $v_{6}$ and $v_{7}$, then $v_{5}$ is adjacent to any one of $\left\{v_{8}, v_{9}\right\}$. Hence no graph exists.

If $v_{3}$ is adjacent to $v_{6}$ and $v_{8}$, then $v_{5}$ is adjacent to any one of $\left\{v_{8}, v_{7}\right\}$ or $v_{9}$. In both cases no graph exists.

If $v_{3}$ is adjacent to $v_{7}$ and $v_{8}$, then $v_{5}$ is adjacent to $v_{8}$ or $v_{9}$. If $v_{5}$ is adjacent to $v_{8}$, then $v_{6}$ is adjacent to $v_{9}$ and hence no graph exists. If $v_{5}$ is adjacent to $v_{9}$, then $v_{6}$ is adjacent to $v_{8}$ and $v_{9}$. Hence $G \cong G_{6}$.

If $v_{3}$ is adjacent to $v_{8}$ and $v_{9}$, then $v_{5}$ is adjacent to any one of $\left\{v_{7}, v_{8}, v_{9}\right\}$. If $v_{5}$ is adjacent to $v_{7}$, then $v_{6}$ is adjacent to $v_{8}$ and $v_{9}$. Hence $G \cong G_{7}$.

Case 2 Let $v_{1}$ be adjacent to $v_{6}$. Then $v_{2}$ is adjacent to any one of $\left\{v_{4}, v_{5}\right\}$ or $\left\{v_{7}, v_{8}, v_{9}\right\}$ or $v_{6}$. If $v_{2}$ is adjacent to $v_{6}$, then $\langle V-S\rangle$ is disconnected and hence no graph exists.

Subcase 1 Let $v_{2} v_{4} \in E(G)$

Let $v_{3}$ be adjacent to $v_{5}, v_{6}$ or $v_{5}, v_{7}$ or $v_{6}, v_{7}$ or $v_{7}, v_{8}$ or $v_{7}, v_{9}$. 
If $v_{3}$ is adjacent to $v_{5}$ and $v_{6}$, then $\langle V-S\rangle$ is disconnected and hence no graph exists. If $v_{3}$ is adjacent to $v_{5}$ and $v_{7}$, then $v_{6}$ is adjacent to any one of $\left\{v_{7}, v_{8}, v_{9}\right\}$. Hence no graph exists.

If $v_{3}$ is adjacent to $v_{6}$ and $v_{7}$, then $v_{5}$ is adjacent to any one of $\left\{v_{7}, v_{8}, v_{9}\right\}$. In this case, no graph exists. If $v_{3}$ is adjacent to $v_{7}$ and $v_{8}$, then $v_{5}$ is adjacent to any one of $\left\{v_{7}, v_{8}, v_{9}\right\}$ and hence no graph exists.

Subcase 2 Let $v_{2} v_{7} \in E(G)$

Let $v_{3}$ be adjacent to both of $\left\{v_{5}, v_{4}\right\}$ or $\left\{v_{4}, v_{6}\right\}$ or $\left\{v_{4}, v_{7}\right\}$ or $\left\{v_{4}, v_{8}\right\}$ or $\left\{v_{7}, v_{6}\right\}$ or $\left\{v_{6}, v_{8}\right\}$.

If $v_{3}$ is adjacent to $v_{4}$ and $v_{5}$, then $\langle V-S\rangle$ is disconnected and hence no graph exists. If $v_{3}$ is adjacent to $v_{4}$ and $v_{6}$, then $v_{5}$ is adjacent to any one of $\left\{v_{7}, v_{8}, v_{9}\right\}$. In all the cases, no graph exists.

If $v_{3}$ is adjacent to $v_{4}$ and $v_{7}$, then $v_{5}$ is adjacent to $v_{8}$ or $v_{9}$. If $v_{5}$ is adjacent to $v_{8}$, then $v_{6}$ is adjacent to $v_{9}$ or $v_{8}$ and hence no graph exists.

If $v_{3}$ is adjacent to $v_{4}$ and $v_{8}$, then $v_{5}$ is adjacent to any one of $\left\{v_{7}, v_{8}, v_{9}\right\}$ and hence no graph exists for this case. If $v_{3}$ is adjacent to $v_{6}$ and $v_{7}$, then $\langle V-S\rangle$ is disconnected and hence no graph exists.

If $v_{3}$ is adjacent to $v_{6}$ and $v_{8}$, then $v_{4}$ is adjacent to $v_{7}$ or $v_{9}$. If $v_{4}$ is adjacent to $v_{7}$, then $v_{5}$ is adjacent to $v_{8}$ and hence no graph exists. If $v_{4}$ is adjacent to $v_{8}$, then $v_{5}$ is adjacent to any one of $\left\{v_{7}, v_{8}, v_{9}\right\}$. In all the above cases, no graph exists.

Case 3 Let $v_{1}$ be adjacent to $v_{7}$. Then $v_{2}$ is adjacent to any one of $\left\{v_{4}, v_{5}\right\}$ or any one of $\left\{v_{8}, v_{9}\right\}$ or $v_{6}$ or $v_{7}$.

Subcase 1 Let $v_{2} v_{4} \in E(G)$

Let $v_{3}$ be adjacent to both of $\left\{v_{5}, v_{6}\right\}$ or $\left\{v_{5}, v_{7}\right\}$ or $\left\{v_{5}, v_{8}\right\}$ or $\left\{v_{6}, v_{7}\right\}$ or $\left\{v_{6}, v_{8}\right\}$ or $\left\{v_{8}, v_{9}\right\}$.

If $v_{3}$ is adjacent to $v_{5}$ and $v_{6}$, then $v_{6}$ is adjacent to any one of $\left\{v_{7}, v_{8}, v_{9}\right\}$. Hence no graph exists. If $v_{3}$ is adjacent to $v_{5}$ and $v_{7}$, then in this case $\langle V-S\rangle$ is disconnected and hence no graph exists.

If $v_{3}$ is adjacent to $v_{5}$ and $v_{8}$, then $v_{6}$ is adjacent to any two of $\left\{v_{7}, v_{8}, v_{9}\right\}$. Hence no graph exists. If $v_{3}$ is adjacent to $v_{6}$ and $v_{7}$, then no graph exists. If $v_{3}$ is adjacent to $v_{6}$ and $v_{8}$, then no graph exists in this case.

If $v_{3}$ is adjacent to $v_{9}$ and $v_{8}$, then $v_{5}$ is adjacent to any one of $\left\{v_{7}, v_{8}, v_{9}\right\}$. If $v_{5}$ is adjacent to $v_{7}$, then $v_{6}$ is adjacent to $v_{9}$ and $v_{8}$. Hence $\langle V-S\rangle$ is disconnected and no graph exists. If $v_{5}$ is adjacent to $v_{8}$, then $v_{6}$ is adjacent to $v_{9}$ and $v_{7}$. Hence $G \cong G_{8}$.

Subcase 2 Let $v_{2} v_{8} \in E(G)$

Let $v_{3}$ be adjacent to both of $\left\{v_{4}, v_{5}\right\}$ or $\left\{v_{4}, v_{6}\right\}$ or $\left\{v_{4}, v_{7}\right\}$ or $\left\{v_{4}, v_{9}\right\}$ or $\left\{v_{6}, v_{7}\right\}$ or $\left\{v_{6}, v_{9}\right\}$ or $\left\{v_{7}, v_{8}\right\}$ or $\left\{v_{7}, v_{9}\right\}$.

If $v_{3}$ is adjacent to $v_{4}$ and $v_{5}$, then in this case $\langle V-S\rangle$ is disconnected and hence no graph exists. If $v_{3}$ is adjacent to $v_{4}$ and $v_{6}$, then no graph exists in this case. 
If $v_{3}$ is adjacent to $v_{4}$ and $v_{5}$, then $v_{5}$ cannot be adjacent to $v_{8}$. Therefore $v_{5}$ is adjacent to $v_{9}$ and $v_{6}$ is adjacent to $v_{8}, v_{9}$. Hence $G \cong G_{9}$.

If $v_{3}$ is adjacent to $v_{4}$ and $v_{9}$, then $v_{5}$ is adjacent to any one of $\left\{v_{7}, v_{8}, v_{9}\right\}$. If $v_{5}$ is adjacent to $v_{7}$, then $v_{6}$ is adjacent to $v_{8}$ and $v_{9}$. Hence $G \cong G_{10}$.

If $v_{3}$ is adjacent to $v_{6}$ and $v_{7}$, then $v_{4}$ is adjacent to $v_{8}$ or $v_{9}$. If $v_{4}$ is adjacent to $v_{8}$, then $v_{9}$ is adjacent to $v_{5}$ and $v_{6}$. Hence $G \cong G_{9}$. If $v_{4}$ is adjacent to $v_{9}$, then $v_{5}$ is adjacent to $v_{8}$ and $v_{6}$ is adjacent to $v_{9}$. Hence $G \cong G_{9}$.

If $v_{3}$ is adjacent to $v_{6}$ and $v_{9}$, then $v_{4}$ is adjacent to any one of $\left\{v_{7}, v_{8}, v_{9}\right\}$. If $v_{4}$ is adjacent to $v_{7}$, then $v_{5}$ is adjacent to $v_{8}$ and $v_{6}$ is adjacent to $v_{9}$. Hence $G \cong G_{11}$.

If $v_{3}$ is adjacent to $v_{7}$ and $v_{8}$, then $\langle V-S\rangle$ is disconnected and hence no graph exists. If $v_{3}$ is adjacent to $v_{7}$ and $v_{9}$, then $v_{4}$ is adjacent to $v_{8}$ and $v_{5}$ is adjacent to $v_{9}$ and hence no graph exists in this case.

Subcase 3 Let $v_{2} v_{6} \in E(G)$

Let $v_{3}$ be adjacent to both of $\left\{v_{4}, v_{5}\right\}$ or $\left\{v_{4}, v_{6}\right\}$ or $\left\{v_{4}, v_{7}\right\}$ or $\left\{v_{4}, v_{8}\right\}$ or $\left\{v_{6}, v_{7}\right\}$ or $\left\{v_{6}, v_{8}\right\}$ or $\left\{v_{7}, v_{8}\right\}$ or $\left\{v_{8}, v_{9}\right\}$.

If $v_{3}$ is adjacent to $v_{4}$ and $v_{5}$, then $\langle V-S\rangle$ is disconnected and hence no graph exists. If $v_{3}$ is adjacent to $v_{4}$ and $v_{6}$, then $v_{5}$ is adjacent to any one of $\left\{v_{8}, v_{9}\right\}$ or $v_{7}$. In both cases, no graph exists.

If $v_{3}$ is adjacent to $v_{4}$ and $v_{7}$, then $v_{5}$ is adjacent to any one of $\left\{v_{8}, v_{9}\right\}$. If $v_{5}$ is adjacent to $v_{8}$, then no graph exists.

If $v_{3}$ is adjacent to $v_{4}$ and $v_{8}$, then $v_{5}$ is adjacent to any one of $\left\{v_{8}, v_{7}\right\}$ or $v_{9}$. In both cases no graph exists. If $v_{3}$ is adjacent to $v_{6}$ and $v_{7}$, then in this case $<V-S>$ is disconnected and hence no graph exists.

If $v_{3}$ is adjacent to $v_{6}$ and $v_{8}$, then $v_{4}$ is adjacent to any one of $\left\{v_{8}, v_{7}\right\}$ or $v_{9}$. In both cases no graph exists.

If $v_{3}$ is adjacent to $v_{7}$ and $v_{8}$, then $v_{4}$ is adjacent to $v_{8}$ or $v_{9}$. If $v_{4}$ is adjacent to $v_{8}$, then $v_{9}$ is adjacent to $v_{5}$ and $v_{6}$. Hence $G \cong G_{12}$. If $v_{4}$ is adjacent to $v_{9}$, then $v_{5}$ is adjacent to $v_{8}$ and $v_{6}$ is adjacent to $v_{9}$. Hence $G \cong G_{12}$.

If $v_{3}$ is adjacent to $v_{9}$ and $v_{8}$, then $v_{4}$ is adjacent to any one of $\left\{v_{7}, v_{8}, v_{9}\right\}$. If $v_{4}$ is adjacent to $v_{7}$, then $v_{5}$ is adjacent to any one of $\left\{v_{8}, v_{9}\right\}$. Without loss of generality, let $v_{5}$ be adjacent to $v_{8}$ and $v_{6}$ be adjacent to $v_{9}$. Hence $G \cong G_{13}$.

Subcase $3 v_{2} v_{7} \in E(G)$

Let $v_{2}$ be adjacent to $v_{7}$. In this case, $\langle V-S\rangle$ is disconnected and hence no graph exists.

Prepositon 2.8. If $\left\langle S>=\bar{K}_{3}\right.$ and $<S_{1}>=<S_{2}>=<S_{3}>=K_{2} \cup K_{1}$, then $G \cong G_{i}$, where $15 \leq i \leq 21$.

Proof. Let $\left.<S_{1}\right\rangle=K_{2} \cup K_{1}=\left(v_{1}, v_{2}, v_{3}\right),\left\langle S_{2}\right\rangle=K_{2} \cup K_{1}=\left(v_{4}, v_{5}, v_{6}\right)$ and $\left.<S_{3}\right\rangle=K_{2} \cup K_{1}=\left(v_{7}, v_{8}, v_{9}\right)$ and $v_{1} v_{2}, v_{4} v_{5}, v_{7} v_{8} \in E(G)$. Let $v_{1}$ be adjacent to any one of $\left\{v_{4}, v_{5}, v_{7}, v_{8}\right\}$ or $\left\{v_{6}, v_{9}\right\}$.

Case 1 Let $v_{1}$ be adjacent to $v_{4}$. Then $v_{2}$ is adjacent to any one of $\left\{v_{7}, v_{8}\right\}$ or $v_{5}$ or $v_{6}$ or $v_{9}$.

Subcase 1 Let $v_{2} v_{7} \in E(G)$ 
Let $v_{3}$ be adjacent to both of $\left\{v_{5}, v_{6}\right\}$ or $\left\{v_{5}, v_{8}\right\}$ or $\left\{v_{6}, v_{9}\right\}$. If $v_{3}$ is adjacent to $v_{5}$ and $v_{6}$, then no graph exists. If $v_{3}$ is adjacent to $v_{5}$ and $v_{8}$, then no graph exists.

If $v_{3}$ is adjacent to $v_{6}$ and $v_{9}$, then $v_{5}$ is adjacent to $v_{8}$ or $v_{9}$. If $v_{5}$ is adjacent to $v_{8}$, then $v_{6}$ is adjacent to $v_{9}$. In this case $\langle V-S\rangle$ is disconnected hence no graph exists. If $v_{5}$ is adjacent to $v_{9}$, then $v_{6}$ is adjacent to $v_{8}$ and hence $G \cong G_{15}$. Subcase 2 Let $v_{2} v_{5} \in E(G)$

In this case, $\langle V-S\rangle$ is disconnected hence no graph exists.

Subcase 3 Let $v_{2} v_{6} \in E(G)$

Let $v_{3}$ be adjacent to both of $\left\{v_{5}, v_{6}\right\}$ or $\left\{v_{5}, v_{7}\right\}$ or $\left\{v_{5}, v_{9}\right\}$ or $\left\{v_{6}, v_{7}\right\}$ or $\left\{v_{6}, v_{9}\right\}$ or $\left\{v_{7}, v_{8}\right\}$ or $\left\{v_{7}, v_{9}\right\}$.

If $v_{3}$ is adjacent to $v_{5}$ and $v_{6}$, then $\langle V-S\rangle$ is disconnected and hence no graph exists. If $v_{3}$ is adjacent to $v_{5}$ and $v_{7}$, then $v_{6}$ must be adjacent to any one of $\left\{v_{7}, v_{8}, v_{9}\right\}$. Hence no graph exists.

If $v_{3}$ is adjacent to $v_{5}$ and $v_{9}$, then $v_{6}$ must be adjacent to any one of $\left\{v_{7}, v_{8}, v_{9}\right\}$. Hence no graph exists. If $v_{3}$ is adjacent to $v_{6}$ and $v_{7}$, then $v_{5}$ must be adjacent to any one of $v_{8}$ or $v_{9}$. In both cases no graph exists.

If $v_{3}$ is adjacent to $v_{6}$ and $v_{9}$, then $v_{5}$ must be adjacent to any one of $\left\{v_{7}, v_{8}, v_{9}\right\}$. Hence no graph exists. If $v_{3}$ is adjacent to $v_{7}$ and $v_{8}$, then $\langle V-S\rangle$ is disconnected and hence no graph exists.

If $v_{3}$ is adjacent to $v_{7}$ and $v_{9}$, then $v_{5}$ is adjacent to $v_{8}$ or $v_{9}$. If $v_{5}$ is adjacent to $v_{8}$ and $v_{6}$ is adjacent to $v_{9}$, then $G \cong G_{17}$. If $v_{5}$ is adjacent to $v_{9}$ and $v_{6}$ is adjacent to $v_{8}$, then $G \cong G_{18}$.

Subcase 4 Let $v_{2} v_{9} \in E(G)$

Let $v_{3}$ be adjacent to both of $\left\{v_{5}, v_{6}\right\}$ or $\left\{v_{5}, v_{7}\right\}$ or $\left\{v_{5}, v_{9}\right\}$ or $\left\{v_{6}, v_{7}\right\}$ or $\left\{v_{6}, v_{9}\right\}$ or $\left\{v_{7}, v_{8}\right\}$ or $\left\{v_{7}, v_{9}\right\}$.

If $v_{3}$ is adjacent to $v_{5}$ and $v_{6}$, then $v_{6}$ is adjacent to any one of $\left\{v_{7}, v_{8}, v_{9}\right\}$ and hence no graph exists. If $v_{3}$ is adjacent to $v_{5}$ and $v_{7}$, then $v_{6}$ is adjacent to $v_{8}$ and $v_{9}$ and hence $G \cong G_{18}$.

If $v_{3}$ is adjacent to $v_{5}$ and $v_{9}$, then $\langle V-S\rangle$ is disconnected and hence no graph exists.

If $v_{3}$ is adjacent to $v_{6}$ and $v_{7}$, then $v_{5}$ is adjacent to any one of $v_{8}$ or $v_{9}$. If $v_{5}$ is adjacent to any one of $v_{8}$, then $v_{6}$ is adjacent to $v_{9}$. Hence $G \cong G_{15}$. If $v_{5}$ is adjacent to any one of $v_{9}$, then $v_{6}$ is adjacent to $v_{8}$. In this case, $\langle V-S\rangle$ is disconnected and hence no graph exists.

If $v_{3}$ is adjacent to $v_{6}$ and $v_{9}$, then $v_{5}$ is adjacent to $v_{7}$ and $v_{6}$ is adjacent to $v_{8}$. Hence $G \cong G_{19}$. If $v_{3}$ is adjacent to $v_{7}$ and $v_{8}$, then $\langle V-S\rangle$ is disconnected and hence no graph exists. If $v_{3}$ is adjacent to $v_{7}$ and $v_{9}$, then no graph exists.

Case 2 Let $v_{1}$ be adjacent to $v_{6}$. Then $v_{2}$ is adjacent to $\left\{v_{4}, v_{5}\right\}$ or $v_{6}$ or $\left\{v_{7}, v_{8}\right\}$ or $v_{9}$. If $v_{2}$ is adjacent to $v_{6}$, then $\langle V-S\rangle$ is disconnected and hence no graph exists in this case.

Subcase 1 Let $v_{2} v_{4} \in E(G)$ 
Let $v_{3}$ be adjacent to both of $\left\{v_{5}, v_{6}\right\}$ or $\left\{v_{5}, v_{7}\right\}$ or $\left\{v_{5}, v_{9}\right\}$ or $\left\{v_{6}, v_{7}\right\}$ or $\left\{v_{6}, v_{9}\right\}$ or $\left\{v_{7}, v_{8}\right\}$ or $\left\{v_{7}, v_{9}\right\}$.

If $v_{3}$ is adjacent to $v_{5}$ and $v_{6}$, then $\langle V-S\rangle$ is disconnected and hence no graph exists in this case. If $v_{3}$ is adjacent to $v_{5}$ and $v_{7}$, then no graph exists. If $v_{3}$ is adjacent to $v_{5}$ and $v_{9}$, then no graph exists.

If $v_{3}$ is adjacent to $v_{6}$ and $v_{7}$, then no graph exists. If $v_{3}$ is adjacent to $v_{6}$ and $v_{9}$, then no graph exists. If $v_{3}$ is adjacent to $v_{8}$ and $v_{7}$, then $\langle V-S\rangle$ is disconnected and hence no graph exists.

If $v_{3}$ is adjacent to $v_{9}$ and $v_{7}$, then $v_{5}$ is adjacent to $v_{8}$ or $v_{9}$. If $v_{5}$ is adjacent to $v_{8}$, then $v_{6}$ is adjacent to $v_{9}$. Hence $G \cong G_{19}$. If $v_{5}$ is adjacent to $v_{9}$, then $v_{6}$ is adjacent to $v_{8}$ and hence $G \cong G_{20}$.

Subcase 2 Let $v_{2} v_{7} \in E(G)$

Let $v_{3}$ be adjacent to both of $\left\{v_{4}, v_{5}\right\}$ or $\left\{v_{4}, v_{6}\right\}$ or $\left\{v_{4}, v_{8}\right\}$ or $\left\{v_{4}, v_{9}\right\}$ or $\left\{v_{6}, v_{8}\right\}$ or $\left\{v_{6}, v_{9}\right\}$ or $\left\{v_{8}, v_{9}\right\}$.

If $v_{3}$ is adjacent to $v_{4}$ and $v_{5}$, then $\langle V-S\rangle$ is disconnected and hence no graph exists. If $v_{3}$ is adjacent to $v_{4}$ and $v_{6}$, then $v_{5}$ is adjacent to $v_{8}$ or $v_{9}$. Hence no graph exists. If $v_{3}$ is adjacent to $v_{4}$ and $v_{8}$, then $v_{9}$ is adjacent to $v_{5}$ or $v_{6}$. Hence $G \cong G_{20}$.

If $v_{3}$ is adjacent to $v_{4}$ and $v_{9}$, then $v_{5}$ is adjacent to $v_{8}$ or $v_{9}$. If $v_{5}$ is adjacent to $v_{8}, v_{6}$ is adjacent to $v_{9}$. Hence $G \cong G_{15}$. If $v_{5}$ is adjacent to $v_{9}$ and $v_{6}$ is adjacent to $v_{8}$, then in this case $\langle V-S\rangle$ is disconnected and hence no graph exists.

If $v_{3}$ is adjacent to $v_{6}$ and $v_{8}$ and $v_{9}$ is adjacent to $v_{4}$ or $v_{5}$, then $\langle V-S\rangle$ is disconnected and hence no graph exists.

If $v_{3}$ is adjacent to $v_{6}$ and $v_{9}$, then $v_{8}$ is adjacent to any one of $\left\{v_{4}, v_{5}\right\}$. If $v_{8}$ is adjacent to $v_{4}$ and $v_{9}$ is adjacent to $v_{5}$, then $G \cong G_{21}$. If $v_{3}$ is adjacent to $v_{8}$ and $v_{9}$, then no graph exists.

Subcase 3 Let $v_{2} v_{9} \in E(G)$

Let $v_{3}$ be adjacent to any two of $\left\{v_{4}, v_{5}, v_{7}, v_{8}\right\}$ or both of $\left\{v_{4}, v_{6}\right\}$ or both of $\left\{v_{6}, v_{9}\right\}$.

If $v_{3}$ is adjacent to $v_{4}$ and $v_{5}$, then $\langle V-S\rangle$ is disconnected and hence no graph exists. If $v_{3}$ is adjacent to $v_{4}$ and $v_{6}$, then no graph exists. If $v_{3}$ is adjacent to $v_{6}$ and $v_{9}$, then $\langle V-S\rangle$ is disconnected and hence no graph exists in this case.

Prepositon 2.9. If $\langle S\rangle=\bar{K}_{3}$ and $<S_{1}>=K_{2} \cup K_{1}$ and $<S_{3}>=<S_{2}>=\bar{K}_{3}$, then $G \cong G_{i}$, where $i=22,23$.

Proof. Let $\left.<S_{1}\right\rangle=K_{2} \cup K_{1}=\left(v_{1}, v_{2}, v_{3}\right),\left\langle S_{2}>=K_{2} \cup K_{1}=\left(v_{4}, v_{5}, v_{6}\right)\right.$ and $<S_{3}>=\bar{K}_{3}=\left(v_{7}, v_{8}, v_{9}\right)$, where $v_{1} v_{2} \in E(G)$. Let $v_{1}$ be adjacent to any one of $\left\{v_{4}, v_{5}, v_{6}, v_{7}, v_{8}, v_{9}\right\}$.

If $v_{1}$ is adjacent to $v_{4}$, then $v_{2}$ is adjacent to any one of $\left\{v_{5}, v_{6}\right\}$ or any one of $\left\{v_{7}, v_{8}, v_{9}\right\}$ or $v_{4}$. If $v_{2}$ is adjacent to $v_{4}$, then in this case $\langle V-S\rangle$ is disconnected and hence no graph exists. 
Case 1 If $v_{2}$ is adjacent to $v_{5}$, then $v_{3}$ is adjacent to both of $\left\{v_{4}, v_{5}\right\}$ or $\left\{v_{4}, v_{6}\right\}$ or $\left\{v_{4}, v_{7}\right\}$ or $\left\{v_{6}, v_{7}\right\}$ or $\left\{v_{7}, v_{8}\right\}$.

If $v_{3}$ is adjacent to $v_{4}$ and $v_{5}$, then $\langle V-S\rangle$ is disconnected and hence no graph exists. If $v_{3}$ is adjacent to $v_{4}$ and $v_{6}$, then no graph exists in this case.

If $v_{3}$ is adjacent to $v_{4}$ and $v_{7}$, then $v_{5}$ is adjacent to any one of $\left\{v_{8}, v_{9}\right\}$ or $v_{7}$. If $v_{5}$ is adjacent to $v_{7}$, then no graph exists. If $v_{5}$ is adjacent to $v_{8}$, then no graph exists.

If $v_{3}$ is adjacent to $v_{6}$ and $v_{7}$, then $v_{4}$ is adjacent to $v_{7}$ or any one of $\left\{v_{8}, v_{9}\right\}$. If $v_{4}$ is adjacent to $v_{7}$, then $v_{5}$ is adjacent to $v_{8}$ and $v_{6}$ is adjacent to $v_{9}$. Hence no graph exists. If $v_{4}$ is adjacent to $v_{8}$, then $v_{5}$ is adjacent to any one of $\left\{v_{7}, v_{8}\right\}$ or $v_{9}$. In both cases no graph exists.

If $v_{3}$ is adjacent to $v_{7}$ and $v_{8}$, then $v_{4}$ is adjacent to any one of $v_{9}$ or $\left\{v_{7}, v_{8}\right\}$. If $v_{4}$ is adjacent to $v_{7}$, then $v_{5}$ is adjacent to $v_{8}$ or $v_{9}$. If $v_{5}$ is adjacent to $v_{8}$, then no graph exists. If $v_{5}$ is adjacent to $v_{9}$, then $v_{6}$ is adjacent to $v_{8}$ or $v_{9}$. Hence $G \cong G_{22}$.

If $v_{4}$ is adjacent to $v_{9}$, then $v_{5}$ is adjacent to any one of $\left\{v_{7}, v_{8}, v_{9}\right\}$. If $v_{5}$ is adjacent to $v_{7}$, then $v_{6}$ is adjacent to $v_{8}$ and $v_{9}$, then $G \cong G_{23}$.

\section{Case 2}

If $v_{2}$ is adjacent to $v_{7}$, then $v_{3}$ is adjacent to both of $\left\{v_{4}, v_{7}\right\}$ or $\left\{v_{4}, v_{5}\right\}$ or $\left\{v_{5}, v_{6}\right\}$. If $v_{3}$ is adjacent to $v_{4}$ and $v_{7}$, then $\langle V-S\rangle$ is disconnected and hence no graph exists.

If $v_{3}$ is adjacent to $v_{4}$ and $v_{5}$, then $v_{5}$ is adjacent to $v_{7}$ or any one of $\left\{v_{8}, v_{9}\right\}$. If $v_{5}$ is adjacent to $v_{7}$, then $\langle V-S\rangle$ is disconnected and hence no graph exists. If $v_{5}$ is adjacent to $v_{8}$, then no graph exists.

If $v_{3}$ is adjacent to $v_{5}$ and $v_{6}$, then $v_{4}$ is adjacent to $v_{7}$ or any one of $\left\{v_{8}, v_{9}\right\}$. If $v_{4}$ is adjacent to $v_{7}$, then $\langle V-S\rangle$ is disconnected and hence no graph exists. If $v_{4}$ is adjacent to $v_{8}$, then no graph exists.

Prepositon 2.10. If $\left\langle S>=<S_{1}>=<S_{2}>=<S_{3}>=\bar{K}_{3}\right.$, then $G \cong G_{i}$, where $24 \leq i \leq 32$.

Proof. Let $\left\langle S_{1}\right\rangle=\bar{K}_{3}=\left(v_{1}, v_{2}, v_{3}\right),\left\langle S_{2}\right\rangle=\bar{K}_{3}=\left(v_{4}, v_{5}, v_{6}\right)$ and $\left\langle S_{3}\right\rangle=$ $\bar{K}_{3}=\left(v_{7}, v_{8}, v_{9}\right)$. Let $v_{1}$ be adjacent to any one of $\left\{v_{4}, v_{5}\right\}$ or any one of $\left\{v_{6}, v_{7}\right\}$.

Case 1 Let $v_{1}$ be adjacent to $v_{4}$ and $v_{5}$. Then $v_{2}$ is adjacent to both of $\left\{v_{4}, v_{5}\right\}$ or $\left\{v_{4}, v_{6}\right\}$ or $\left\{v_{4}, v_{7}\right\}$ or $\left\{v_{6}, v_{7}\right\}$ or $\left\{v_{7}, v_{8}\right\}$.

Subcase $1 v_{2} v_{4}, v_{2} v_{5} \in E(G)$

Let $v_{2}$ be adjacent to $v_{4}$ and $v_{5}$. In this case, $\langle V-S\rangle$ is disconnected and hence no graph exists.

Subcase $2 v_{2} v_{4}, v_{2} v_{6} \in E(G)$

If $v_{2}$ is adjacent to $v_{4}$ and $v_{6}$, then $v_{3}$ is adjacent to both of $\left\{v_{5}, v_{6}\right\}$ or $\left\{v_{5}, v_{7}\right\}$ or $\left\{v_{7}, v_{8}\right\}$. If $v_{3}$ is adjacent to $v_{5}$ and $v_{6}$, then $\langle V-S\rangle$ is disconnected and hence no graph exists. If $v_{3}$ is adjacent to $v_{5}$ and $v_{7}$, then no graph exists. If $v_{3}$ is adjacent to $v_{8}$ and $v_{7}$, then no graph exists.

Subcase $3 v_{2} v_{4}, v_{2} v_{7} \in E(G)$ 
Let $v_{3}$ be adjacent to both of $\left\{v_{6}, v_{5}\right\}$ or $\left\{v_{5}, v_{7}\right\}$ or $\left\{v_{5}, v_{8}\right\}$ or $\left\{v_{6}, v_{7}\right\}$ or $\left\{v_{6}, v_{8}\right\}$ or $\left\{v_{7}, v_{8}\right\}$ or $\left\{v_{8}, v_{9}\right\}$.

If $v_{3}$ is adjacent to $v_{5}$ and $v_{6}$, then no graph exists. If $v_{3}$ is adjacent to $v_{5}$ and $v_{7}$, then $\langle V-S\rangle$ is disconnected and hence no graph exists.

If $v_{3}$ is adjacent to $v_{5}$ and $v_{8}$, then no graph exists. If $v_{3}$ is adjacent to $v_{6}$ and $v_{7}$, then no graph exists. If $v_{3}$ is adjacent to $v_{6}$ and $v_{8}$, then no graph exists.

If $v_{3}$ is adjacent to $v_{7}$ and $v_{8}$. Since $\mathrm{G}$ is cubic, $v_{5}$ cannot be adjacent to $v_{8}$. Hence $v_{5}$ is adjacent to $v_{9}$ and $v_{6}$ is adjacent to $v_{9}$ and $v_{8}$. Hence $G \cong G_{24}$.

If $v_{3}$ is adjacent to $v_{9}$ and $v_{8}$, then $v_{5}$ is adjacent to $v_{7}$ and $v_{6}$ is adjacent to $v_{8}$ and $v_{9}$. In this case, $\langle V-S\rangle$ is disconnected and hence no graph exists.

Subcase $4 v_{2} v_{6}, v_{2} v_{7} \in E(G)$

Let $v_{3}$ be adjacent to both of $\left\{v_{4}, v_{5}\right\}$ or $\left\{v_{4}, v_{7}\right\}$ or $\left\{v_{7}, v_{8}\right\}$ or $\left\{v_{8}, v_{9}\right\}$.

If $v_{3}$ is adjacent to $v_{4}$ and $v_{5}$, then $\langle V-S\rangle$ is disconnected and hence no graph exists. If $v_{3}$ is adjacent to $v_{4}$ and $v_{7}$, then no graph exists.

If $v_{3}$ is adjacent to $v_{7}$ and $v_{8}$, then $v_{4}$ must be adjacent to $v_{8}$ or $v_{9}$. If $v_{4}$ is adjacent to $v_{8}$, then $v_{9}$ is adjacent to $v_{5}$ and $v_{6}$. Hence $G \cong G_{25}$. If $v_{4}$ is adjacent to $v_{9}$, then $v_{5}$ is adjacent to $v_{8}$ and $v_{6}$. Hence $G \cong G_{26}$.

If $v_{3}$ is adjacent to $v_{9}$ and $v_{8}$, then $v_{4}$ must be adjacent to $v_{7}, v_{5}$ is adjacent to $v_{8}$ and $v_{6}$ is adjacent to $v_{9}$. Hence $G \cong G_{27}$.

Subcase $5 v_{2} v_{7}, v_{2} v_{8} \in E(G)$

Let $v_{3}$ be adjacent to both of $\left\{v_{4}, v_{5}\right\}$ or $\left\{v_{4}, v_{6}\right\}$ or $\left\{v_{4}, v_{7}\right\}$ or $\left\{v_{6}, v_{9}\right\}$.

If $v_{3}$ is adjacent to $v_{4}$ and $v_{5}$, then $\langle V-S\rangle$ is disconnected and hence no graph exists. If $v_{3}$ is adjacent to $v_{4}$ and $v_{6}$, then no graph exists.

Let $v_{3}$ is adjacent to $v_{4}$ and $v_{7}$. Since $\mathrm{G}$ is cubic, $v_{5}$ cannot be adjacent to $v_{8}$. If $v_{5}$ is adjacent to $v_{9}$, then $v_{6}$ is adjacent to $v_{8}$ and $v_{9}$. Hence $G \cong G_{24}$.

If $v_{3}$ is adjacent to $v_{6}$ and $v_{9}$, then $v_{4}, v_{5}, v_{6}$ are adjacent to $v_{7}, v_{8}, v_{9}$. Hence $<V-S>$ is disconnected and hence no graph exists.

Case 2 If $v_{1}$ is adjacent to $v_{6}$ and $v_{7}$, then $v_{2}$ is adjacent to both of $\left\{v_{4}, v_{5}\right\}$ or $\left\{v_{4}, v_{6}\right\}$ or $\left\{v_{6}, v_{7}\right\}$ or $\left\{v_{6}, v_{8}\right\}$. If $v_{2}$ is adjacent to $v_{6}$ and $v_{7}$, then $\langle V-S\rangle$ is disconnected and hence no graph exists.

Subcase $1 v_{2} v_{4}, v_{2} v_{5} \in E(G)$

Let $v_{3}$ be adjacent to both of $\left\{v_{4}, v_{5}\right\}$ or $\left\{v_{4}, v_{7}\right\}$ or $\left\{v_{4}, v_{8}\right\}$ or $\left\{v_{7}, v_{8}\right\}$ or $\left\{v_{8}, v_{9}\right\}$.

If $v_{3}$ is adjacent to $v_{4}$ and $v_{5}$, then $\langle V-S\rangle$ is disconnected and hence no graph exists. If $v_{3}$ is adjacent to $v_{4}$ and $v_{7}$, then no graph exists.

If $v_{3}$ is adjacent to $v_{4}$ and $v_{8}$, then no graph exists. If $v_{3}$ is adjacent to $v_{7}$ and $v_{8}$, then $v_{4}$ is adjacent to $v_{8}$ and $v_{9}$ is adjacent to $v_{5}$ and $v_{6}$. Hence $G \cong G_{28}$.

If $v_{3}$ is adjacent to $v_{9}$ and $v_{8}$, then $v_{4}$ is adjacent to $v_{7}$ and $v_{5}$ is adjacent to $v_{8}$ and $v_{6}$. Hence $G \cong G_{29}$.

Subcase: $2 v_{2} v_{4}, v_{2} v_{6} \in E(G)$

Let $v_{3}$ be adjacent to both of $\left\{v_{4}, v_{5}\right\}$ or $\left\{v_{4}, v_{7}\right\}$ or $\left\{v_{4}, v_{8}\right\}$ or $\left\{v_{5}, v_{7}\right\}$ or $\left\{v_{8}, v_{5}\right\}$ or $\left\{v_{7}, v_{8}\right\}$ or $\left\{v_{8}, v_{9}\right\}$. 
If $v_{3}$ is adjacent to $v_{4}$ and $v_{5}$, then no graph exists. If $v_{3}$ is adjacent to $v_{4}$ and $v_{7}$, then $\langle V-S\rangle$ is disconnected and hence no graph exists. If $v_{3}$ is adjacent to $v_{4}$ and $v_{8}$, then no graph exists.

If $v_{3}$ is adjacent to $v_{7}$ and $v_{5}$, then no graph exists. If $v_{3}$ is adjacent to $v_{8}$ and $v_{5}$, then no graph exists. Let $v_{3}$ be adjacent to $v_{7}$ and $v_{8}$. Since $\mathrm{G}$ is cubic $v_{4}$ cannot be adjacent to $v_{8}$. Hence $v_{4}$ is adjacent to $v_{9}$ and $v_{5}$ is adjacent to $v_{8}, v_{9}$. Hence $G \cong G_{24}$.

If $v_{3}$ is adjacent to $v_{8}$ and $v_{9}$, then $v_{4}$ is adjacent to $v_{7}, v_{5}$ is adjacent to $v_{8}$ and $v_{9}$. Hence $G \cong G_{30}$.

Subcase: $3 v_{2} v_{6}, v_{2} v_{8} \in E(G)$

Let $v_{3}$ be adjacent to both of $\left\{v_{4}, v_{5}\right\}$ or $\left\{v_{4}, v_{7}\right\}$ or $\left\{v_{4}, v_{9}\right\}$ or $\left\{v_{7}, v_{8}\right\}$ or $\left\{v_{7}, v_{9}\right\}$.

If $v_{3}$ is adjacent to $v_{4}$ and $v_{5}$, then no graph exists. Let $v_{3}$ be adjacent to $v_{4}$ and $v_{7}$. Since $\mathrm{G}$ is cubic, $v_{4}$ cannot be adjacent to $v_{8}$. Hence $v_{4}$ is adjacent to $v_{9}$ and $v_{5}$ is adjacent to $v_{8}$ and $v_{9}$. Hence $G \cong G_{31}$.

If $v_{3}$ is adjacent to $v_{4}$ and $v_{9}$, then $v_{4}$ is adjacent to $v_{8}$ and $v_{9}$. Hence $G \cong G_{32}$. If $v_{3}$ is adjacent to $v_{7}$ and $v_{8}$, then no graph exists. If $v_{3}$ is adjacent to $v_{7}$ and $v_{9}$, then no graph exists.

Theorem 2.1. Let $G$ be a 3 -regular graph of order twelve. Then $\chi(G)=\gamma_{[1,2] c c}(G)=$ 3 if and only if $G \cong G_{i}$, where $1 \leq i \leq 32$.

Proof. If $\mathrm{G}$ is any one of the graphs $G_{i}$, where $1 \leq i \leq 32$ as in the figure 1 , then clearly verified that $\chi(G)=\gamma_{[1,2] c c}(G)=3$. Conversly, assume that $\chi(G)=$ $\gamma_{[1,2] c c}(G)=3$. Then the proof follows from proposition 2.1 to 2.10 .

Conclusion. In this paper we investigated 3-regular graphs of order 12, whose $[1,2]$-Complementary connected domination and chromatic number are eqaual to three.

\section{REFERENCES}

[1] Chartrand, G.and Lesniak, L., Graphs and Digraphs, Fourth Edition CRC Press, Boca Raton, 2005.

[2] Haynes, T.W., Hedetniemi, S.T. and Slater, P.J., Fundamentals of Domination in Graphs, Marcel Dekker, Inc., New York, 1998.

[3] Chellali, Mustapha, Haynes, Teresa W., Hedetniemi, Stephen T. and McRae, Alice, [1, 2]-Sets in graphs, Discrete Applied Mathematics, 161, (2013), 2885 - 2893.

[4] Mahadevan, G., Renuka, K. and Sivagnanam, C., [1,2]-Complementary connected domination number of graphs, International Journal of Computational and Applied Mathematics, 12(1), (2017), $281-288$.

[5] Mahadevan, G., Renuka, K. and Sivagnanam, C., [1,2]-Complementary connected domination number of graphs-II, International Journal of Pure and Applied Mathematics, 117(11), (2017), $165-175$.

[6] Kulli, V.R. and Janakiraman, B., The nonsplit domination number of a graph, Indian Journal of Pure and Applied Math, Vol. 31, No. 5, (2000), 545 - 550.

[7] Yang, Xiaojing and Wu, Baoyindureng, [1,2]-domination in graphs, Discrete Applied Mathematics, 175, (2014), $79-86$. 
Current address: G.Mahadevan: Department of Mathematics, The Gandhigram Rural Institute - Deemed to be University, Gandhigram, Dindigul-624302, India.

E-mail address: drgmaha2014@gmail.com

ORCID Address: http://orcid.org/0000-0002-1471-1626

Current address: K.Renuka: Department of Mathematics, Department of Science and Humanities, Vignan University, Andhra Pradesh, India

E-mail address: math.renuka@gmail.com

ORCID Address: http://orcid.org/0000-0001-7573-2191 